\title{
Double regularization medical CT image blind restoration reconstruction based on proximal alternating direction method of multipliers
}

\author{
Jingyu Zhang ${ }^{1}$, Yunshan Sun ${ }^{2^{*}}$, Yuan Zhang ${ }^{1}$ and Jianfu Teng ${ }^{1}$
}

\begin{abstract}
To solve the problem of $\mathrm{CT}$ image degradation, a double regularization $\mathrm{CT}$ image blind restoration reconstruction method was proposed. The objective function including both a clear image and point spread function was established. To avoid the over-smoothing phenomenon and protect the detail, the objective function includes two constraint regularization terms. They are total variation (TV) and wavelet sparsity respectively. The objective function was solved by the alternating direction multiplier method (ADMM), and the optimal solution was obtained. Firstly, the CT image blind restoration reconstruction was decomposed into two sub-problems: reconstructed image estimation and point spread function estimation. Furthermore, each sub-problem can be solved by the proximal alternating direction method of multipliers. Finally, the CT image blind restoration reconstruction was realized. The experimental results show that the proposed algorithm takes into account the degradation effect of projection data, and the proposed algorithm is superior to other existing algorithms in the subjective visual effect. At the same time, in the aspect of objective evaluation, the proposed algorithm improves the objective image quality metrics such as peak signal-to-noise ratio (PSNR), structural similarity index metric (SSIM), and universal image quality index (UIQI).
\end{abstract}

Keywords: Blind restoration, Image reconstruction, CT image, Proximal alternating direction method of multipliers

\section{Introduction}

Computer tomography $(\mathrm{CT})$ is that $\mathrm{X}$-ray beam is used to scan some certain thickness parts of the body. X-ray through the detection layer is received by the detector. CT reflects the degree of organ and tissue absorption of X-ray. It is conducive to find small lesions in any part of the body. CT can greatly improve the early detection rate and pathological diagnosis rate. CT detection has become one of the most important diagnostic basis in the field of medical image diagnosis. However, in the imaging and transmission process, CT image will be affected by many factors, such as electronic radiation detection effect of defocus blur, detector number and spacing, frame geometry,

\footnotetext{
* Correspondence: zhangjinyu_8825@163.com

${ }^{2}$ School of Information Engineering, Tianjin University of Commerce, Tianjin, China

Full list of author information is available at the end of the article
}

reconstruction algorithm and other factors. A point of human tissue can be mapped to many points on CT image. In other words, each point on CT image is a mixture of many points of human tissue. And its correspondence is generally referred to as a point spread function, that is, the transmission characteristics of the whole system. Image restoration can be achieved by measuring the point spread function of CT system to eliminate the effect of degradation $[1,2]$.

Medical CT image blind restoration algorithm use only CT degradation image of the CT system to estimate the point spread function. Then, the purpose of image restoration is achieved and the measurement of the point spread function is avoided. Jiang et al. [3] firstly proposed the concept of medical CT blind restoration. They used the expectation maximization strategy to estimate point spread function and image restoration. And, they gave the 
assumption that the degradation function of CT system has Gaussian distribution. Multiple sets of bone interfaces and the iterative strategy [4-6] were utilized to estimate the point spread function. Blind equalization algorithm was applied to the two-dimensional CT blind image restoration [7-9]. Combining with the dimension reduction theory and orthogonal transform, without any information of degradation, only the features of degraded images were utilized to estimate the clear image. In addition, there are special uses of image restoration technology to eliminate specific CT artifacts [10-13]. In general, in the process of CT imaging, there is always the degradation. CT image degradation or artifact was eliminated by the use of specialized techniques, but it is bound to increase the complexity of the algorithm. If we can consider the influence of the projection data degradation and overcome it, it will improve the quality of the reconstructed image.

Total variational (TV) is a very important optimization constraint in CT reconstruction and was widely studied and applied. Candes et al. [14] proposed a compressive sensing theory. This theory proved that sparse signals can be accurately restored by data far less than the sampling theorem, which greatly facilitates the research and application of TV constraints in CT reconstruction, especially sparse projection data CT reconstruction [15-18]. Hu et al. [19] proposed a reconstruction method that the pre-scan CT images or analytic reconstruction images were used as the iterative initial conditions to improve the speed of TV constraint reconstruction. In the whole variation method, the assumption that the image segmentation smoothing will cause the small edge of the CT image and the texture of the texture are blurred, resulting in the "smooth" phenomenon, there is also the problem of slow reconstruction. In order to solve the above problems, the researchers also combine some sparse constraints with the TV method, or directly use sparse constraints. Wang et al. [20] studied a TV-based optimization model for Micro-CT reconstruction based on an upsampling of the reconstruction grid with original detector and X-ray dose. Using an extension of the gradient projection method, an alternating minimization algorithm is employed to solve the corresponding energy function. Lu-zhen et al. [21] proposed a hybrid compression-aware reconstruction method based on the total variation and non-aliasing contourlet transform. The method uses non-aliasing sontourlet transform as a sparse representation of CT image. The objection function is solved by splitting the Bregman algorithm. In a word, it is helpful to improve the effect of image reconstruction by adding proper regularization term. Chen et al. [22] proposed image domain artifacts used in low-dose CT image suppression dictionary learning method. The establishment of sparse representation of the discriminant dictionary filters a part of the artifacts and noise. And then, the general dictionary learning was used to further reduce noise and residual artifacts.

Alternating direction multiplier method (ADMM) is a simple and effective method for solving separable convex programming problems, especially on solving large-scale problems. In the ADMM algorithm, the objective function of the original problem is divided into several subproblems which are easy to find local solutions, and the global solution of the original problem is obtained. It is also applied in the reconstruction of the compression perception. Ramani et al. [23] used the method of variable separation to separate the shift and shift invariant in the statistical data model and established two equivalent constraint problems. Then, the objection function is solved by the alternating direction multiplier methods. Sawatzky et al. [24] established a multi-channel framework for the reconstruction of medical CT images and optimized the solution by the alternating direction multiplier method.

At present, CT image reconstruction algorithm often does not consider the influence of CT system point spread function, but this assumption is unreasonable. CT image is often affected by the system point spread function in the imaging process. The point spread function results in image degradation [25]. In this paper, a new blind reconstruction model of medical CT image is established. Considering the influence of sampling data by degradation factors in image reconstruction, the proposed method avoids the elimination of point spread function alone. So it is beneficial to improve the quality of reconstructed image, reduce the complexity of the algorithm, and improve the ability of real-time processing.

\section{Method}

\subsection{Proximal alternating direction method of multipliers for solving unconstrained optimization problems}

Suppose an unconstrained optimization problem as follows:

$$
\min _{\mathbf{x} \in \Re^{n}} f(\mathbf{x})+g(\mathbf{G x})
$$

where $f: \mathfrak{R}^{n} \rightarrow(-\infty,+\infty)$ and $g: \mathfrak{R}^{m} \rightarrow(-\infty,+\infty)$ are closed convex function and $\mathbf{G} \in \mathfrak{R}^{m \times n}$ is a transformation matrix.

ADMM is an effective method to solve formula (1), which combines the advantages of multiplier method and alternating minimization method. This method introduces dummy variables $\mathbf{u}$ and $\mathbf{d}$. And then, an iterative solution is obtained by the idea of alternating minimization, that is,

$$
\mathbf{x}_{k+1} \leftarrow \arg \min _{\mathbf{x}} f(\mathbf{x})+\frac{\mu}{2}\left\|\mathbf{G x}-\mathbf{u}_{k}-\mathbf{d}_{k}\right\|_{2}^{2}
$$

where $\mu$ is a constant. 


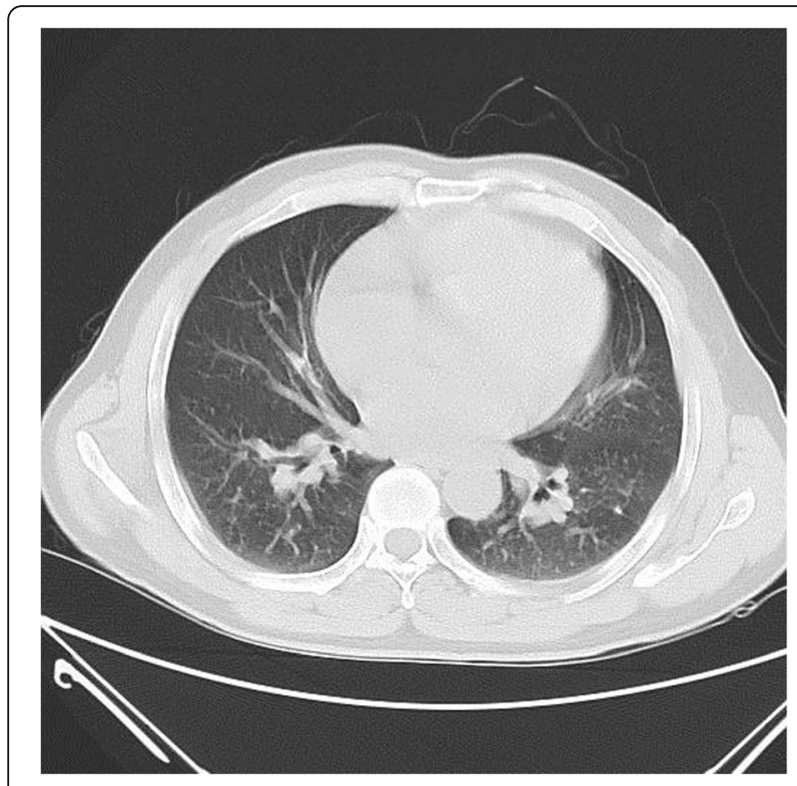

Fig. 1 CT phantom image

$$
\begin{aligned}
& \mathbf{u}_{k+1} \leftarrow \arg \min _{\mathbf{u}} g(\mathbf{u})+\frac{\mu}{2}\left\|\mathbf{G} \mathbf{x}_{k+1}-\mathbf{u}-\mathbf{d}_{k}\right\|_{2}^{2} \\
& \mathbf{d}_{k+1} \leftarrow \mathbf{d}_{k}-\left(\mathbf{G x}_{k+1}-\mathbf{u}_{k+1}\right)
\end{aligned}
$$

Formulas (2)-(4) are iterated to obtain the optimal solution formula (1). The feasibility and convergence of ADMM has been demonstrated.

To improve the application of ADMM in the image processing problem, here considering a generic unconstrained optimization problem:

$$
\min _{\mathbf{x} \in \Re^{n}} f(\mathbf{x})+\sum_{i=1}^{J} g_{i}\left(\mathbf{H}^{(i)} \mathbf{x}\right)
$$

where $g_{i}(\cdot)$ is a closed convex function. $\mathbf{H}^{(j)} \in \mathfrak{R}^{m_{j} \times n}$ is a random matrix, and $m=m_{1}+m_{2}+\cdots+m_{\text {J }}$. Definition $\mathbf{G}$ $=\left[\mathbf{H}^{(1)}, \mathbf{H}^{(2)}, \cdots, \mathbf{H}^{()}\right]^{*} \in \mathfrak{R}^{m \times n}, \quad$ represents a conjugate transposition of matrix or a vector.

In image reconstruction, assume $f(\mathbf{x})=0$, formula (2) becomes a quadratic optimization problem in this case. The parameters $\mu$ in formula (2) affect the convergence speed. In the sub-optimization problem, different weight coefficients are used to improve the ability of the algorithm, that is, the quadratic optimization problem becomes

$\mathbf{x}_{k+1} \leftarrow \arg \min _{\mathbf{x}}(\mathbf{G x}-\mathbf{u}-\mathbf{d})^{*} \mathbf{Y}(\mathbf{G x}-\mathbf{u}-\mathbf{d})=\arg \min _{\mathbf{x}}(\mathbf{G x}-\boldsymbol{\zeta})^{*} \mathbf{Y}(\mathbf{G x}-\boldsymbol{\zeta})$

Here, $\Upsilon=\operatorname{diag}(\underbrace{\mu_{1}, \cdots, \mu_{1}}_{m_{1} \text { elements }}, \cdots, \underbrace{\mu_{j}, \cdots, \mu_{j}}_{m_{j} \text { elements }}, \cdots, \underbrace{\mu_{J}, \cdots, \mu_{J}}_{m_{J} \text { elements }})$.

The quadratic problem can be done using the gradient method $(\mathbf{G x}-\boldsymbol{\zeta})^{*} \mathbf{\Upsilon}(\mathbf{G x}-\boldsymbol{\zeta})$ to solve, getting

$\mathbf{x}_{k+1} \leftarrow\left(\mathbf{G}^{*} \mathbf{Y} \mathbf{G}\right)^{-1} \mathbf{G}^{*} \boldsymbol{\Upsilon} \zeta=\left[\sum_{j=1}^{J} \mu_{j}\left(\mathbf{H}^{(j)}\right)^{*} \mathbf{H}^{(j)}\right]^{-1} \sum_{j=1}^{J} \mu_{j}\left(\mathbf{H}^{(j)}\right)^{*} \zeta^{(j)}$

where $\boldsymbol{\zeta}^{(j)}=\mathbf{u}_{k}^{(j)}+\mathbf{d}_{k}^{(j)}, \quad \boldsymbol{\zeta}^{(j)}, \quad \mathbf{u}_{k}^{(j)}$ with $\mathbf{d}_{k}^{(j)}(j=1, \cdots, J)$ are respectively sub-vectors of $\zeta, \mathbf{u}_{k}$, and $\mathbf{d}_{k}$.

In formula (5), assume

$$
g(\mathbf{u})=\sum_{j=1}^{J} g_{j}\left(\mathbf{u}^{(j)}\right)
$$

where $\mathbf{u}^{(j)} \in \boldsymbol{R}^{m_{j}}$ is a sub-vector of $\mathbf{u}, \mathbf{u}=\left[\left(\mathbf{u}^{(1)}\right)^{*}\right.$, $\left.\cdots,\left(\mathbf{u}^{(/)}\right)^{*}\right]^{*}$.

Solving the iterative optimization, formula (8) is decomposed into $J$-independent sub-optimization problems. Sub-optimization problem is solved by the proximity operator. Different optimization problems require different proximity operators. As can be seen, formula (8) is transformed into $J$ proximity operators to solve, which is

$$
\mathbf{u}_{k+1} \leftarrow \operatorname{prox}_{g / \mu}\left(\mathbf{G x}_{k+1}-\mathbf{d}_{k}\right)
$$

where $\operatorname{prox}(\cdot)$ is a proximity operator. The most frequently used proximity operators include Moreau proximity operator and vector soft approximation operator.

Formula (5) can be optimized by iterating formulas (7), (9), and (4).

\subsection{Double regularization $\mathrm{CT}$ image blind restoration reconstruction}

CT blind image restoration reconstruction model represents as follows:
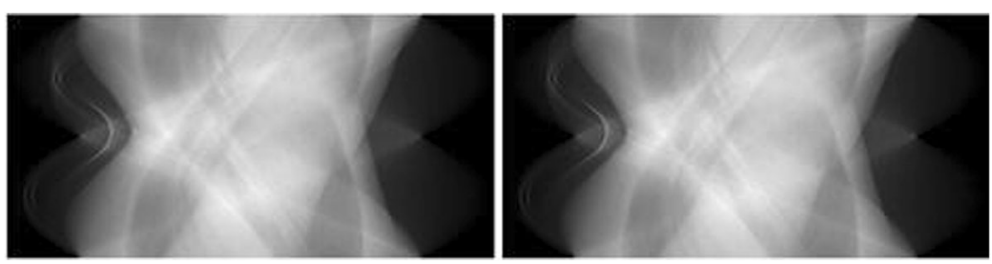

Fig. 2 Image of projection data 


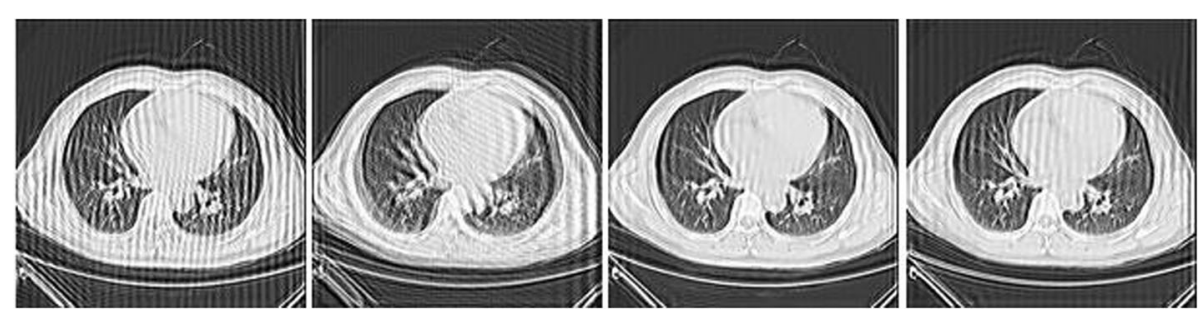

Fig. 3 Comparison of reconstructed images with different reconstruction methods (SNR $=20 \mathrm{~dB}$ )

$$
\mathbf{P}=\mathbf{A H}_{p s f} \mathbf{x}+\mathbf{n}
$$

where $\mathbf{P}$ represents the measured projection data of $\mathrm{CT}$. A represents the sampling matrix of the system. $\mathbf{H}_{p s f}$ represents an unknown image degradation matrix. $\mathbf{x}$ indicates that a clear image. $\mathbf{n}$ is the noise vector introduced in the measurement.

CT image reconstruction is the process that a clear image is reconstructed in the case of a given projection data $\mathbf{P}$. In order to ensure reconstruction quality, two or more regularization terms were used to solve unconstrained optimization problem. An iterative formula is utilized to achieve the CT image reconstruction. Then, it is possible to accurately reconstruct $\mathrm{X}$ from $\mathbf{P}=\mathbf{A H}_{p s} \mathbf{X}+\mathbf{n}$. In practical applications, in order to improve the quality of reconstruction, some regularization terms are usually used in image reconstruction. In this way, an ill-posed problem is transformed into a well-posed problem. This will guarantee the existence, uniqueness, and stability of the solution. It is helpful to obtain a relatively stable and meaningful solution from the meaning of the solution. Total variation (TV) transformation is a kind of commonly used regularization prior model. For example, reference [15] used TV minimization constraint for cone beam CT reconstruction. However, for low-contrast images, the method sometimes results in loss of information. In order to overcome this shortcoming, a new image reconstruction method is proposed in this paper. The method uses two kinds of regularization terms: the total variation transformation and the sparse transformation. Sparse transform has two advantages. First, it is very good to maintain the edge structure. Second, it has a protective effect on low-contrast information. The total variation transform can effectively suppress noise interference and strip artifacts. The model proposed in this paper can achieve a good balance between artifact suppression and detail protection. In CT image reconstruction, the image does not necessarily satisfy the sparsity. Therefore, the good sparse transform can ensure that the image sparse representation has a good sparsity. In other words, when $\boldsymbol{\alpha}=\Theta \mathbf{x}$, the image representation $\boldsymbol{\alpha}$ is more sparse than the original image $\mathbf{x} . \Theta$ represents the sparse wavelet transform.

The problem of blind restoration of double regularization $\mathrm{CT}$ images under incomplete projection data can be achieved by the following unconstrained minimization objective function.

$\hat{\mathbf{x}}=\arg \min _{\mathbf{x}} \frac{\mu}{2}\left\|\mathbf{P}-\mathbf{A} \mathbf{H}_{p s f} \mathbf{x}\right\|_{2}^{2}+\lambda_{1}\|\boldsymbol{\Theta} \mathbf{x}\|_{1}+\lambda_{2} \sum_{i=1}^{n}\left\|\mathbf{D}_{i} \mathbf{x}\right\| 2$

Among them, $\mathbf{D}_{i}$ is used to calculate the difference between the horizontal and vertical directions of the $i^{\text {th }}$ pixel.

The first term in formula (11) represents the difference between the projection of the reconstructed projection and the measured projection, that is, the data fidelity item; the second term is the wavelet sparse transformation constraint term; the third term is the total variation difference constraint term.

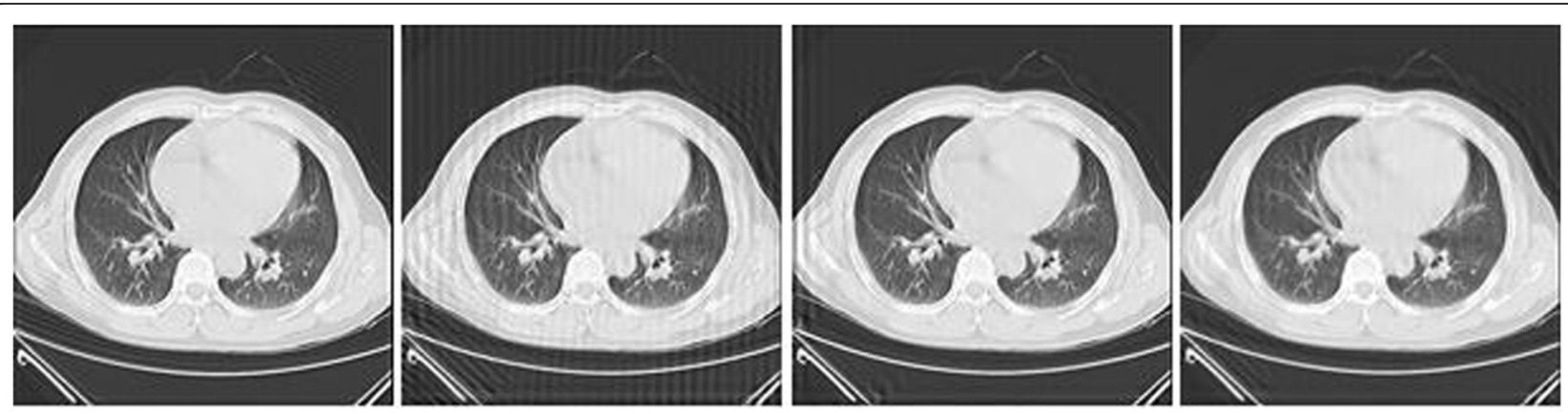

Fig. 4 Comparison of reconstructed images with different reconstruction methods ( $S N R=40 \mathrm{~dB})$ 
Table 1 Comparison of image evaluation parameters of different reconstruction algorithms $(S N R=20 \mathrm{~dB})$

\begin{tabular}{lllll}
\hline & FBP & SART & TV regularization & The proposed method \\
\hline PSNR & 56.4641 & 56.5765 & 57.5832 & 58.1515 \\
SSIM & 0.9954 & 0.9961 & 0.9968 & 0.9976 \\
UIQI & 0.0172 & 0.0163 & 0.0164 & 0.0506 \\
\hline
\end{tabular}

\subsection{ADMM method for solving double regularized CT} image blind rebuild reconstruction

Reconstruction of CT image blind restoration can be divided into image restoration reconstruction sub-problem and degraded function estimation sub-problem. To solve one of both variables, the other one can be regarded as a constant amount.

The purpose of CT image blind reconstruction is to solve formula (11). ADMM was used to solve the optimization problem. Formula (11) is converted into formula (5), as follows

$$
\begin{gathered}
g_{1}\left(\mathbf{u}^{(1)}\right)=\left\|\mathbf{P}-\mathbf{u}^{(1)}\right\|_{2}^{2} \\
g_{2}\left(\mathbf{u}^{(2)}\right)=\lambda_{1}\left\|\mathbf{u}^{(2)}\right\|_{1} \\
g_{j}\left(\mathbf{u}^{(j)}\right)=\lambda_{2}\left\|\mathbf{u}^{(j)}\right\|_{2}, \text { Among them, } j=3,4 \\
\mathbf{H}^{(1)} \in \mathfrak{R}^{l \times n}, \quad \mathbf{H}^{(1)}=\mathbf{A} \mathbf{H}_{p s f} \\
\mathbf{H}^{(2)} \in \Re^{d \times n}, \quad \mathbf{H}^{(2)}=\boldsymbol{\Theta} \\
\mathbf{H}^{(j)} \in \mathfrak{R}^{2 \times n}, \quad \mathbf{H}^{(j)}=\mathbf{D}_{j-2} \text { Among them }, j=3,4
\end{gathered}
$$

Formulas (12)-(17) are taken into formula (7) and let $\mu_{3}=\mu_{4}=\mu$, then

$$
\begin{aligned}
\sum_{j=1}^{J} \mu_{j}\left(\mathbf{H}^{(j)}\right)^{*} \mathbf{H}^{(j)} & =\mu_{1} \mathbf{H}_{p s f}^{*} \mathbf{A}^{*} \mathbf{A} \mathbf{H}_{p s f}+\mu_{2} \boldsymbol{\Theta}^{*} \boldsymbol{\Theta}+\mu \sum_{j=3}^{n+2} \mathbf{D}_{j-2}^{*} \mathbf{D}_{j-2} \\
& =\mu_{1} \mathbf{H}_{p s f}^{*} \mathbf{A}^{*} \mathbf{A} \mathbf{H}_{p s f}+\mu_{2} \boldsymbol{\Theta}^{*} \boldsymbol{\Theta}+\mu\left[\left(\mathbf{D}^{h}\right)^{*} \mathbf{D}^{h}+\left(\mathbf{D}^{v}\right)^{*} \mathbf{D}^{\nu}\right] \\
& =\mu_{1} \mathbf{H}_{p s f}^{*} \mathbf{A}^{*} \mathbf{A} \mathbf{H}_{p s f}+\mu_{2} \boldsymbol{\Theta}^{*} \boldsymbol{\Theta}+\mu \mathbf{D}^{*} \mathbf{D}
\end{aligned}
$$

Among them, (.)* represents a conjugate transpose matrix. $\mathbf{D}=\left[\begin{array}{l}\mathbf{D}^{h} \\ \mathbf{D}^{v}\end{array}\right], \quad \mathbf{D}_{1}=\mathbf{D}^{h}$ calculates the difference of the image in the horizontal direction, and $\mathbf{D}_{2}=\mathbf{D}^{v}$ calculates the difference in the vertical direction.

Table 2 Comparison of image evaluation parameters of different reconstruction algorithms $(S N R=40 \mathrm{~dB})$

\begin{tabular}{lllll}
\hline & FBP & SART & TV regularization & The proposed method \\
\hline PSNR & 58.6194 & 59.9776 & 60.0533 & 61.6325 \\
SSIM & 0.9982 & 0.9991 & 0.9992 & 0.9996 \\
UIQI & 0.0152 & 0.0570 & 0.0832 & 0.1565 \\
\hline
\end{tabular}

Table 3 Comparison of image evaluation parameters of different reconstruction algorithms (head CT)

\begin{tabular}{lllll}
\hline & FBP & SART & TV regularization & The proposed method \\
\hline PSNR & 58.6891 & 58.8119 & 58.9634 & 61.8236 \\
SSIM & 0.9966 & 0.9967 & 0.9983 & 0.9993 \\
UIQI & 0.1830 & 0.1357 & 0.046 & 0.0296 \\
\hline
\end{tabular}

$\mathbf{H}_{p s f}^{*} \mathbf{A}^{*} \mathbf{A} \mathbf{H}_{p s f}$ is a diagonal matrix. $\alpha_{i j}$ represents any element of the matrix. $\forall \alpha_{i j} \alpha_{j i} \geq 0$ and $\mathbf{x} \geq 0$, so the data $\left\|\mathbf{P}-\mathbf{A H}_{p s f} \mathbf{x}\right\|_{2}^{2}$ in formula (11) is a positive definite quadratic function.

For ease of calculation, a periodic boundary difference operator that can be diagonalized by Fourier transform is

$$
\begin{aligned}
& \mathbf{D}^{h}=\mathbf{F}^{*} \boldsymbol{\Delta}^{h} \mathbf{F} \\
& \mathbf{D}^{v}=\mathbf{F}^{*} \boldsymbol{\Delta}^{v} \mathbf{F}
\end{aligned}
$$

Among them, $\mathbf{F}$ is a two-dimensional discrete Fourier transform, $\mathbf{F}$ is a unitary matrix, $\Delta^{h}$, and $\Delta^{v}$ are diagonal matrix.

Thus, formula (21) can be rewritten as

$$
\begin{aligned}
\sum_{j=1}^{4} \mu_{j}\left(\mathbf{H}^{(j)}\right)^{*} \mathbf{H}^{(j)} & =\mu_{1} \mathbf{F} \mathbf{H}_{p s f}^{*} \mathbf{A}^{*} \mathbf{A} \mathbf{H}_{p s f}+\mu_{2} \boldsymbol{\Theta}^{*} \boldsymbol{\Theta}+\mu \sum_{j=3}^{4} \mathbf{D}_{j-2}^{*} \mathbf{D}_{j-2} \\
& =\mu_{1} \mathbf{H}_{p s f}^{*} \mathbf{A}^{*} \mathbf{A} \mathbf{H}_{p s f}+\mu_{2} \boldsymbol{\Theta}^{*} \boldsymbol{\Theta}+\mu\left[\left(\mathbf{D}^{h}\right)^{*} \mathbf{D}^{h}+\left(\mathbf{D}^{v}\right)^{*} \mathbf{D}^{v}\right] \\
& =\mu_{1} \mathbf{H}_{p s f}^{*} \mathbf{A}^{*} \mathbf{A} \mathbf{H}_{p s f}+\mu_{2} \boldsymbol{\Theta}^{*} \boldsymbol{\Theta}+\mu\left[\mathbf{F}^{*} \Delta^{h} \mathbf{F} \mathbf{F}^{*} \Delta^{h} \mathbf{F}+\mathbf{F}^{*} \boldsymbol{\Delta}^{v} \mathbf{F} \mathbf{F}^{*} \boldsymbol{\Delta}^{v} \mathbf{F}\right] \\
& =\mu_{1} \mathbf{H}_{p s f}^{*} \mathbf{A}^{*} \mathbf{A} \mathbf{H}_{p s f}+\mu_{2} \boldsymbol{\Theta}^{*} \boldsymbol{\Theta}+\mu\left[\mathbf{F}^{*} \Delta^{h} \Delta^{h} \mathbf{F}+\mathbf{F}^{*} \boldsymbol{\Delta}^{v} \boldsymbol{\Delta}^{v} \mathbf{F}\right]
\end{aligned}
$$

The quadratic problem in the algorithm is solved by the gradient method

$\mathbf{x}_{k+1} \leftarrow \mathbf{K}\left[\mu_{1} \mathbf{H}_{p s f}^{*} \mathbf{A}^{*}\left(\mathbf{u}_{k}^{(1)}+\mathbf{d}_{k}^{(1)}\right)+\mu_{2} \boldsymbol{\Theta}^{*}\left(\mathbf{u}_{k}^{(2)}+\mathbf{d}_{k}^{(2)}\right)+\mu \sum_{j=3}^{4} \mathbf{D}_{j-2}^{*}\left(\mathbf{u}_{k}^{(j)}+\mathbf{d}_{k}^{(j)}\right)\right]$

where, $\mathbf{K}=\left[\mu_{1} \mathbf{H}_{p s f}^{*} \mathbf{A}^{*} \mathbf{A} \mathbf{H}_{p s f}+\mu_{2} \boldsymbol{\Theta}^{*} \boldsymbol{\Theta}+\mu \sum_{j=3}^{4} \mathbf{D}_{j-2}^{*} \mathbf{D}_{j-2}\right]^{-1}$.

For optimization problems in different forms, different proximity operators are required. For the $\mathbf{u}_{k+1}^{(1)}$, the essence is to solve a quadratic programming problem, you can use the gradient method to get

$$
\begin{aligned}
\mathbf{u}_{k+1}^{(1)} & =\operatorname{prox}_{g_{1} / \mu_{1}}\left(\mathbf{A} \mathbf{H}_{p s f} \mathbf{x}_{k+1}-\mathbf{d}_{k}^{(1)}\right) \\
& =\arg \min _{\mathbf{u}} \frac{1}{\mu_{1}}\|\mathbf{y}-\mathbf{u}\|_{2}^{2}+\frac{1}{2}\left\|\mathbf{u}-\left(\mathbf{A} \mathbf{H}_{p s f} \mathbf{x}_{k+1}-\mathbf{d}_{k}^{(1)}\right)\right\|_{2}^{2} \\
& =\frac{2}{2+\mu_{1}}\left(\mathbf{y}+\frac{\mu_{1}}{2}\left(\mathbf{A} \mathbf{H}_{p s f} \mathbf{x}_{k+1}-\mathbf{d}_{k}^{(1)}\right)\right)
\end{aligned}
$$

In order to solve $\ell_{1}$ norm, the soft threshold function as a proximity operator is usually used, which is 


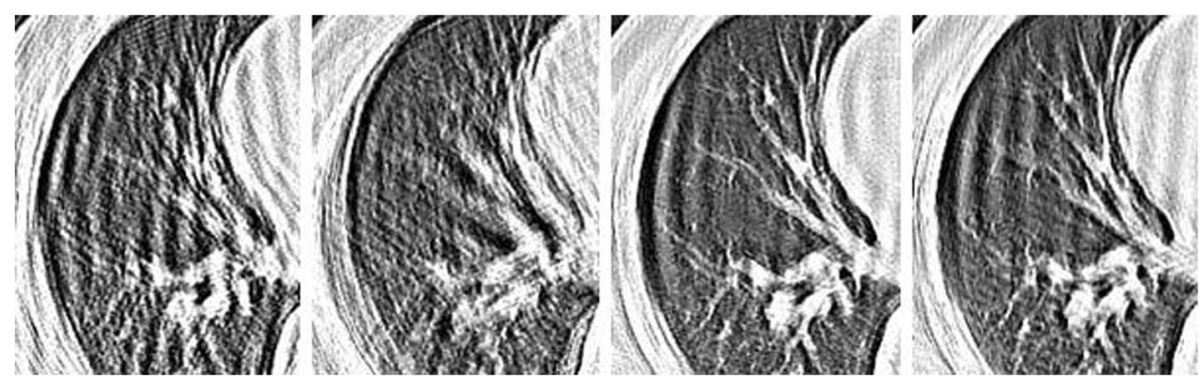

Fig. 5 Comparison of reconstructed images of local details amplification with different reconstruction methods (SNR $=20 \mathrm{~dB})$

$$
\operatorname{soft}(\mathbf{v}, \tau)=\operatorname{sign}(\mathbf{v}) \otimes \max \{|\mathbf{v}|-\tau, 0\}
$$

Among them, $\otimes$ represents the multiplication of the corresponding element.

$$
\begin{aligned}
\mathbf{u}_{k+1}^{(2)} & =\operatorname{prox}_{g_{2} / \mu_{1}}\left(\boldsymbol{\Theta} \mathbf{x}_{k+1}-\mathbf{d}_{k}^{(2)}\right) \\
& =\arg \min _{\mathbf{u}} \frac{\lambda_{1}}{\mu_{2}}\|\mathbf{u}\|_{1}+\frac{1}{2}\left\|\mathbf{u}-\left(\boldsymbol{\Theta} \mathbf{x}_{k+1}-\mathbf{d}_{k}^{(2)}\right)\right\|_{2}^{2} \\
& =\operatorname{sof} t\left(\boldsymbol{\Theta} \mathbf{x}_{k+1}-\mathbf{d}_{k}^{(2)}, \frac{\lambda_{1}}{\mu_{2}}\right)
\end{aligned}
$$

Among them, $\operatorname{soft}(\cdot)$ is a soft threshold function.

$$
\begin{aligned}
\mathbf{u}_{k+1}^{(j)} & =\operatorname{prox}_{g_{j} / \mu}\left(\mathbf{D}_{j-2}^{*} \mathbf{x}_{k+1}-\mathbf{d}_{k^{(j)}}\right) \\
& =\arg \min _{\mathbf{u}} \frac{\lambda_{2}}{\mu}\|\mathbf{u}\|_{2}+\frac{1}{2}\left\|\mathbf{u}-\left(\mathbf{D}_{j-2}^{*} \mathbf{x}_{k+1}-\mathbf{d}_{k}^{(j)}\right)\right\|_{2}^{2} \\
& =\text { vector-soft }\left(\mathbf{D}_{j-2}^{*} \mathbf{x}_{k+1}-\mathbf{d}_{k}^{(j)}, \frac{\lambda_{2}}{\mu}\right) \quad j=3,4
\end{aligned}
$$

Among them, vector- $\operatorname{soft}(\cdot)$ is vector soft threshold function. vector $-\operatorname{soft}(\mathbf{v}, \lambda)=\frac{\mathbf{v}}{\|\mathbf{v}\|_{2}} \max \left(\|\mathbf{v}\|_{2}-\lambda, 0\right)$.

The iterative relationship of the introduced intermediate variable $\mathbf{d}$ is as follows:

$$
\begin{aligned}
& \mathbf{d}_{k+1}^{(1)}=\mathbf{d}_{k}^{(1)}-\left(\mathbf{R F}_{p} \mathbf{x}_{k+1}-\mathbf{u}_{k+1}^{(1)}\right) \\
& \mathbf{d}_{k+1}^{(2)}=\mathbf{d}_{k}^{(2)}-\left(\boldsymbol{\Theta} \mathbf{x}_{k+1}-\mathbf{u}_{k+1}^{(2)}\right) \\
& \mathbf{d}_{k+1}^{(j)}=\mathbf{d}_{k}^{(j)}-\left(\mathbf{D}_{j-2}^{*} \mathbf{x}_{k+1}-\mathbf{u}_{k+1}^{(j)}\right) \quad j=3,4
\end{aligned}
$$

The optimal solution of formula (11) can be obtained by iterative algorithm. In other words, we obtain an estimate of the reconstructed image.

For solving the point spread function sub-problems, we always see $\mathbf{x}$ as a constant to solve $\mathbf{H}_{p s f}$. In order to ensure the convergence, we add the total variance paradigm of each element in $\mathbf{H}_{p s f}$ as a constraint term, $\left\|\nabla h_{p s f}\right\|_{1}=\sum \sqrt{h_{p s f, x}^{2}+h_{p s f, y}^{2}}$. Among them, $\Sigma$ represents the superposition of all the elements.

$$
\hat{\mathbf{H}}_{p s f}=\arg \min _{\mathbf{H}_{p s f}} \frac{\mu}{2}\left\|\mathbf{P}-\mathbf{A} \mathbf{H}_{p s f} \mathbf{x}\right\|_{2}^{2}+\gamma\left\|\nabla h_{p s f}\right\|_{1}
$$

As before, the optimal solution of formula (30) can be obtained by ADMM method too.

\section{Results and discussion \\ 3.1 Results}

To verify the effectiveness of the proposed algorithm, human lung CT images were used for the first simulation experiments, and phantom image is $512 \times 512$ pixels, as shown in Fig. 1. In this paper, the fan beam source is
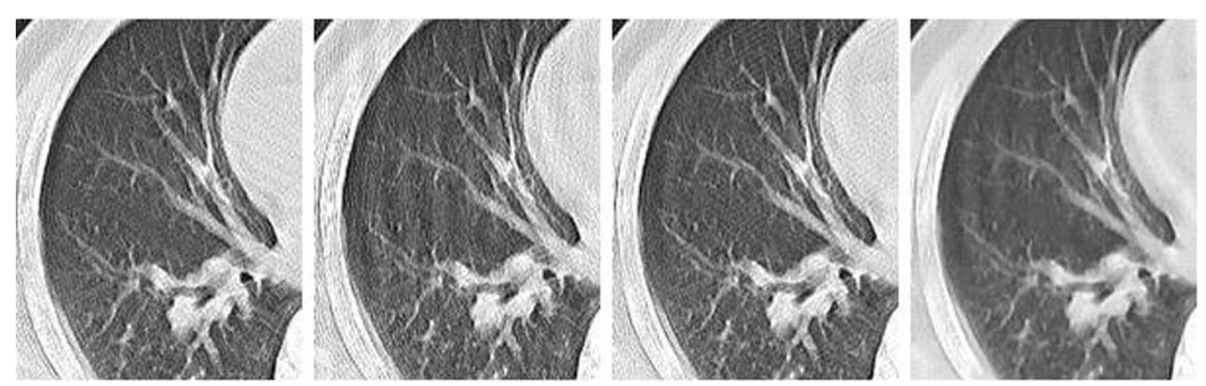

Fig. 6 Comparison of reconstructed images of local details amplification with different reconstruction methods $(S N R=40 \mathrm{~dB})$ 


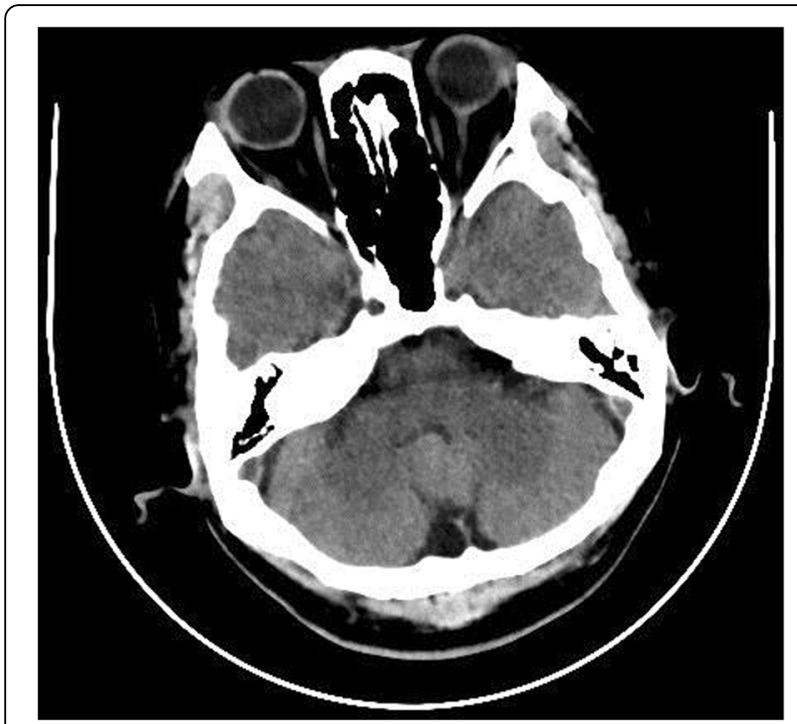

Fig. 7 Head CT phantom image

simulated and the center of the image is coincident with the center of rotation. The projection data was obtained by the uniform sampling in the range of $0 \sim 360$. Each projection data was used by FBP, SART, TV regularization, and the algorithm proposed for image reconstruction. All the reconstruction methods are simulated at the same degradation condition. In the experiment, the degradation process of CT image is approximated by a Gauss point spread function, the degraded image is generated by the Gauss filter of $\sigma=1$, and the Gauss white noise with the noise power of $\sigma_{n}^{2}$ is superimposed. Signal-to-noise ratio (SNR) is defined as SNR $=10 \log _{10}\left(\|\mathbf{x}\|_{2}^{2} / \sigma_{n}^{2}\right)$. The experimental analysis is carried out respectively under the condition of SNR of 20 and $40 \mathrm{~dB}$. The projection data under under different signal-tonoise ratios $20 \mathrm{~dB}$ and $40 \mathrm{~dB}$ is shown in Fig. 2. The selected sparse wavelet transform is db20 wavelet, and the decomposition level is 2 . The iterative algorithm termination condition is $\left\|\mathbf{x}_{k+1}-\mathbf{x}_{k}\right\|_{2}^{2} /\left\|\mathbf{x}_{k+1}\right\|_{2}^{2} \leq 2 \times 10^{-6}$. The maximum number of iteration is set to 50 . On the other hand, in the sub-problem of point spread function estimation, the algorithm termination condition is $\|\mathbf{h}(l+1)-\mathbf{h}(l)\| /\|\mathbf{h}(l)\| \leq 10^{-3}$. The maximum number of iteration is set to 100 .
The experiments were performed in a computer Intel(R) i7-4770@3. 60 GHz with 16 G RAM with MATLAB 2016a programming. The experimental parameters are as follows: $\mu=1, \lambda_{1}=10^{-3} \mu, \lambda_{2}=10^{-4} \mu, \gamma=10^{-3} \mu$. For ADMM parameters, we used heuristic rules, which lead to a good performance of the algorithm. $\mu_{1}$ and $\mu_{2}$ are chosen to be proportional to $\lambda_{2}$ specifically $\mu_{1}=10 \lambda_{2}$ and $\mu_{2}=100 \lambda_{2}$.

From the reconstruction effect pictures that can be seen, FBP algorithm is seriously affected by the noise with the serious artifacts and poor effect. SART algorithm is better than FBP algorithm, but the artifacts are still serious; although there is no obvious streak artifact in TV regularization algorithm, the image is too smooth and the detail is lost. The proposed algorithm in this paper is the best in terms of clarity, contrast, and detail retention. In order to see more clearly the details of the retention effect, a part of Figs. 3 and 4 was taken to enlarge and contrast in this paper, as shown in Figs. 5 and 6. As can be seen from the figure, the method can effectively suppress the noise, preserve the image details, clearly see the small blood vessels, and have good reconstruction effect. The artifacts of FBP and SART algorithm are serious, and the TV algorithm does not have obvious artifacts, but it is too smooth and it lost too many details.

To evaluate the restored images, three metrics, i.e., peak signal-to-noise ratio (PSNR), universal image quality index (UIQI) [26], and structural similarity index metric (SSIM) [27], are employed. Among these metrics, the ideal value of PSNR is $+\infty$, and the ideal values of both UIQI and SSIM are 1. These three metrics can only be used in the simulated experiments because they require the existence of a reference image. The above four metrics tabulated for each experiment are the average values over 10 times repetitions. The results are shown in Tables 1 and 2 .

\subsection{Discussion}

The experimental results show that the structural similarity of image restoration method based on double regularization is increased, and the image quality is improved. The proposed method can obtain better visual effect and improve the PSNR. Compared with other
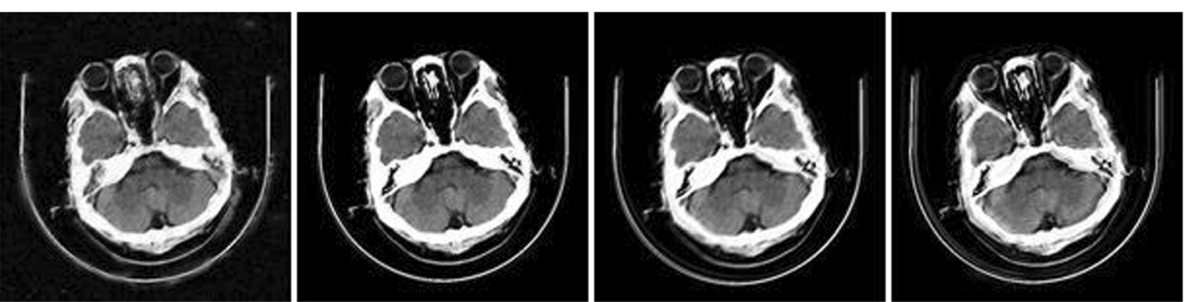

Fig. 8 Comparison of reconstructed images with different reconstruction methods (head CT) 


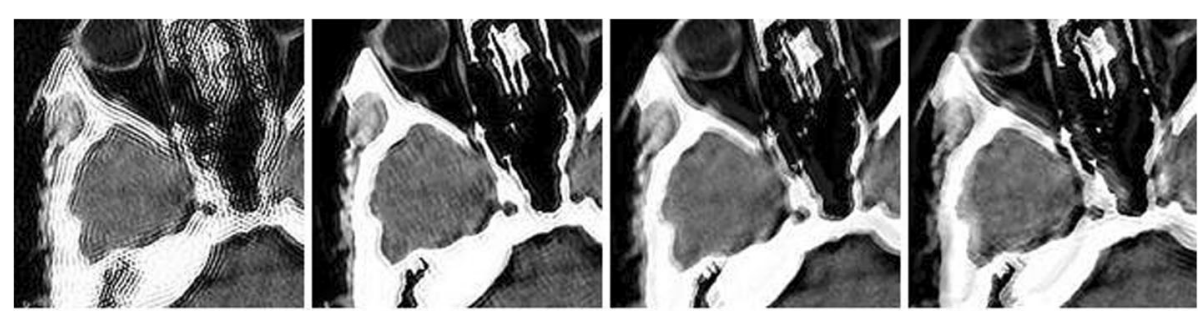

Fig. 9 Comparison of reconstructed images of local details amplification with different reconstruction methods (head CT)

methods, the proposed algorithm has stronger ability to recover details. It can be seen from Tables 1 and 2 that the new algorithm improves the objective image quality metrics such as the PSNR, structural similarity, and UIQI value. At the same time, under different parameters, the new algorithm has a better effect of restoration and reconstruction, showing that the algorithm has better robustness.

In the second simulation, a head CT image is chosen to verify the algorithm performance as shown in Fig. 7 . The image size contains $512 \times 512$ pixels.

For comparison purposes, the projection data is denoised by Hanning filter. In the case of limited-angle projection, the number of projection angle was set 180 . The limited-angle projection was reconstructed by algorithms of by FBP, SART, TV regularization, and the proposed algorithm.

The reconstructed images are shown in Fig. 8. To view the texture more clearly, a part of an image is amplified, as shown in Fig. 9. From the reconstruction figures that can be seen, the reconstructed images by FBP and SART have some artifacts in the case of limited-angle projection. The reconstructed image by TV regularization algorithm does not have obvious stripe-shape artifacts, and the images are too smooth. The proposed algorithm has the best effects such as sharpness, contrast, and detail preservation.

To quantitatively evaluate the effectiveness of the proposed algorithm, the reconstructed images shown in Fig. 8 and the ideal phantom shown in Fig. 7 are compared using SSIM, PSNR, and UIQI, as shown in Table 3.

From Table 3, we can see that PSNR and UIQI are improved compared with the other algorithms. SSIM of the proposed algorithm is much close to 1 . That means the method proposed can get an image that is highly similar to the ideal phantom image.

\section{Conclusions}

An algorithm for double regularization medical CT image blind restoration reconstruction based on proximal alternating direction method of multipliers is proposed. The proposed method combines the total variation and wavelet domain sparsity regularization constraint for image restoration and reconstruction. To solve the large-scale optimization problem in image restoration, an approximate alternating direction multiplier method is used. The experimental results show that, compared with the current image reconstruction algorithm, the proposed algorithm has a better subjective visual effect. In the aspect of objective evaluation, the algorithm proposed in this paper improves the objective image quality metrics such as the peak signal-to-noise ratio, structural similarity index metric, and universal image quality index. At the same time, multiregularization constraint reduces the sensitivity of regularization parameter in a certain range, which is beneficial to the maintenance of the complex edge penalty terms, and preserves the image edges and details.

\section{Acknowledgements \\ The authors thank the editor and anonymous reviewers for their helpful comments and valuable suggestions.}

\section{Funding}

This work was supported by Tianjin Research Program of Application Foundation and Advanced Technology (Nos.16JCYBJC28800 and 13JCYBJC15600), Tianjin Excellent Postdoctoral International Training Program, Tianjin Commerce University Young and Middle-aged Teacher International Training Program.

Availability of data and materials

We can provide the data.

\section{Authors' contributions}

JZ and JT conceived and designed the study. YS and YZ performed the experiments. JZ wrote the paper. JZ, JT, and YS reviewed and edited the manuscript. All authors read and approved the final manuscript.

\section{Competing interests}

The authors declare that they have no competing interests.

\section{Publisher's Note}

Springer Nature remains neutral with regard to jurisdictional claims in published maps and institutional affiliations.

\section{Author details}

${ }^{1}$ School of Electrical and Information Engineering, Tianjin University, Tianjin, China. ${ }^{2}$ School of Information Engineering, Tianjin University of Commerce, Tianjin, China.

Received: 14 July 2017 Accepted: 12 October 2017

Published online: 30 October 2017

References

1. Z Liang, B Kun, H Kuidong, et al., Multi-pinhole imaging measurement and assessment method for PSF of cone-beam CT system. Chin. J. Sci. Instrum. 33(9), 2061-2066 (2012) 
2. A Kayugawa, M Ohkubo, S Wada, Accurate determination of CT point-spreadfunction with high precision. J. Appl. Clin. Med. Phys. 14(4), 216-226 (2013)

3. M Jiang, G Wang, MW Skinner, et al., Blind deblurring of spiral CT images. IEEE Trans. Med. Imaging 22(7), 837-845 (2003)

4. JY Xu, K Taguchi, BMW Tsui, Statistical projection completion in X-ray CT using consistency conditions. IEEE Trans. Med. Imaging 29(8), 1528-1540 (2010)

5. A Pakdel, JG Mainprize, N Robert, et al., Model-based PSF and MTF estimation and validation from skeletal clinical CT images. Med. Phys. 41(1), 8 (2014) Article ID: 011906

6. T Taniguchi, G Akamatsu, Y Kasahara, et al., Improvement in PET/CT image quality in overweight patients with PSF and TOF. Ann. Nucl. Med. 29(1), 71-77 (2015)

7. YS Sun, LY Zhang, J Zhang, et al., Neural network blind equalization algorithm applied in medical $C T$ image restoration. Math. Probl. Eng. 2013(28) Article ID:743546 (2013)

8. YS Sun, LY Zhang, HY Zhang, et al., Multichannel blind CT image restoration via variable splitting and alternating direction method. Method Trans. Tianjin Univ. 21(6), 524-532 (2015)

9. YS Sun, LY Zhang, HY Zhang, Complex system blind equalization algorithm for equivalent multichannel $\mathrm{CT}$ image restoration. J. Med. Imaging Health Informatics 6, 555-561 (2016)

10. GP Chang, $\Pi T$ Chang, TS Pan, et al., Joint correction of respiratory motion artifact and partial volume effect in lung/thoracic PET/CT imaging. Med. Phys. 37(12), 6221-6232 (2010)

11. JH Lewis, R Li, X Jia, et al., Mitigation of motion artifacts in CBCT of lung tumors based on tracked tumor motion during CBCT acquisition. Phys. Med. Biol. 56(17), 5485-5502 (2011)

12. XH Meng, YC Chen, DQ Zheng, Correcting respiratory motion blur in PET images based on phantom and deconvolution algorithm. Chin. J. Biomed. Eng. 32(6), 655-662 (2013)

13. C Mory, V Auvray, B Zhang, et al., Removing streak artifacts from ECG-gated reconstructions using deconvolution. J. Xray. Sci. Technol. 22(2), 253-270 (2014)

14. EJ Candès, JK Romberg, T Tao, Stable signal recovery from incomplete and inaccurate measurements. Commun. Pure Appl. Math. 59(8), 1207-1223 (2006)

15. EY Sidky, CM Kao, X Pan, Accurate image reconstruction from few-views and limited-angle data in divergent-beam CT. J. Xray. Sci. Technol. 14(2), 119-139 (2009)

16. E Sidky, X Pan, Image reconstruction in circular cone-beam computed tomography by constrained, total-variation minimization. Phys. Med. Biol. 53(17), 4777-4807 (2008)

17. LA Vese, Expectation maximization and total variation-based model for computed tomography reconstruction from undersampled data//SPIE medical imaging. Int. Soc. Opt. Photonics, 7961(3),165-177 (2011)

18. J Chen, J Cong, LA Vese, et al., A hybrid architecture for compressive sensing 3-D CT reconstruction. IEEE J. Emerg. Top. Circuits Syst. 2(3), 616-625 (2012)

19. $\mathrm{Z} \mathrm{Hu}, \mathrm{H}$ Zheng, Improved total variation minimization method for few-view computed tomography image reconstruction. Biomed. Eng. Online 13(1), 1-10 (2014)

20. LY Wang, ZH Wei, SH Luo, et al., Image super reconstruction for micro-CT based on compressed sensing. J. Image Graph. 17(4)، 487-493 (2012)

21. L-z Deng, P Feng, M-y Chen, P He, Q-s Vo, B Wei, A CT reconstruction algorithm based on non-aliasing contourlet transform and compressive sensing. Comput. Math. Methods Med. 11, 9 (2014) Article ID: 753615

22. Y Chen, LY Shi, QJ Feng, J Yang, HZ Shu, LM Luo, JL Coatrieux, WF Chen, Artifact suppressed dictionary learning for low-dose CT image processing. IEEE Trans. Med. Imaging 33(12), 2271-2292 (2014)

23. S Ramani, JA Fessler, A splitting-based iterative algorithm for accelerated statistical X-ray CT reconstruction. IEEE Trans. Med. Imaging 31(3), 677-688 (2012)

24. A Sawatzky, QF Xu, CO Schirra, et al., Proximal ADMM for multi-channel image reconstruction in spectral X-ray CT. IEEE Trans. Med. Imaging 33(8), 1657-1688 (2014)

25. $Y \mathrm{Hu}, \mathrm{L} X \mathrm{Xie}, \mathrm{L} L \mathrm{Luo}$, et al., $\mathrm{L} 0$ constrained sparse reconstruction for multi-slice helical CT reconstruction. Phys. Med. Biol. 56(4), 1173-1189 (2011)

26. AYA Yusra, CS Der, Comparison of image quality assessment: PSNR, HVS, SSIM, UIQI. Int. J. Sci. Engineering 3(1), 1-5 (2012)

27. Z Wang, AC Bovik, HR Sheikh, et al., Image quality assessment: from error visibility to structural similarity. IEEE Trans. Image Process. 13(4), 600-612 (2004)

\section{Submit your manuscript to a SpringerOpen ${ }^{\circ}$ journal and benefit from:}

- Convenient online submission

- Rigorous peer review

- Open access: articles freely available online

- High visibility within the field

- Retaining the copyright to your article

Submit your next manuscript at $\gg$ springeropen.com 